UNIVERSIDADE DE SÃO PAULO

ESCOLA DE EDUCAÇÃO FÍSICA E ESPORTE

\title{
MOTIVAÇÃO E GINÁSTICA ARTÍSTICA FORMATIVA NO CONTEXTO EXTRACURRICULAR
}

PRISCILA LOPES

SÃO PAULO

2009 
PRISCILA LOPES

Dissertação apresentada à Escola de Educação Física e Esporte da Universidade de São Paulo, como requisito parcial para obtenção do grau de Mestre em Educação Física.

ORIENTADORA: Prof. ${ }^{a}$ Dr. ${ }^{a}$ MYRIAN NUNOMURA 


\section{AGRADECIMENTOS}

Gostaria de agradecer, primeiramente, aos meus pais, Vera e Toninho, que são os grandes responsáveis por toda minha formação e pela pessoa que sou hoje.

Ao meu marido, Cris, que sempre me incentivou e me apoiou nos momentos difíceis desta interminável jornada.

À toda minha família e amigos que acompanharam todo meu percurso com palavras de apoio, incentivo e carinho.

À minha orientadora Prof. ${ }^{a}$ Dr. ${ }^{a}$ Myrian Nunomura pela oportunidade, pela paciência, pelo apoio sempre seguro em todos os momentos, e por tornar fácil e difícil a tarefa de concluir um mestrado.

À todos do Esporte Clube Pinheiros pelo apoio e compreensão nos momentos em que precisei me ausentar para realização desta pesquisa, e pelo auxílio com questões particulares da Ginástica.

Aos amigos do EUNEGI que me apoiaram e auxiliaram do início ao fim desta pesquisa. Um agradecimento especial à Mari, que sempre se preocupou com meu desenvolvimento acadêmico e profissional.

Aos coordenadores e professores dos colégios participantes desta pesquisa que se disponibilizaram e me auxiliaram com tanto gosto no processo de coleta de dados.

Aos colegas da secretaria de pós-graduação e da biblioteca, agradeço a competência e a ajuda em todos os momentos de dúvidas.

E, finalmente, aos membros da banca. Muito obrigada! 
SUMÁRIO

Página

LISTA DE QUADROS........................................................ vi

LISTA DE FIGURAS................................................. x

LISTA DE SÍGLAS, ABREVIAÇÕES E SÍMBOLOS.................... xix

LISTA DE ANEXOS...............................................................

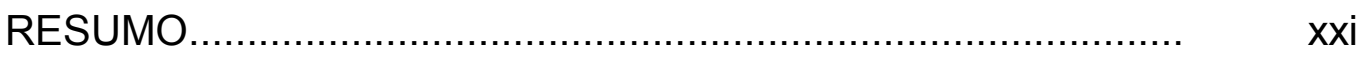

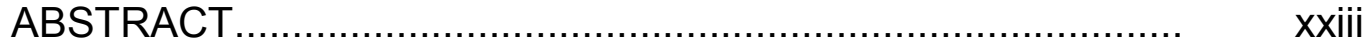

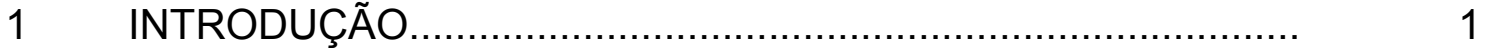

2 REVISÃO DA LITERATURA.....................................................

2.1 A prática de atividade física para crianças e jovens.................... 5

2.2 O Esporte para crianças e jovens.............................................. 9

2.3 A realidade das atividades esportivas extracurriculares nas escolas particulares da Grande são Paulo................................. 16

2.4 Características do praticante................................................... 19

2.5 Conhecendo a Ginástica Artística............................................ 27

2.5.1 Características da prática de Ginástica Artística.......................... 34

2.5.2 A questão da especialização precoce........................................ 37

2.5.3 A Ginástica Artística no contexto das atividades esportivas extracurriculares nas escolas particulares................................. 41

2.5.4 Motivação e Ginástica Artística................................................. 42

2.6 Motivação para a prática de atividade física e esporte................ 49

2.6.1 Definições e conceitos de motivação......................................... 48

2.6.2 Motivação intrínseca e extrínseca............................................. 54

2.6.3 Motivação para iniciar.............................................................. 5

2.6.4 Motivação e aprendizagem...................................................... 64 


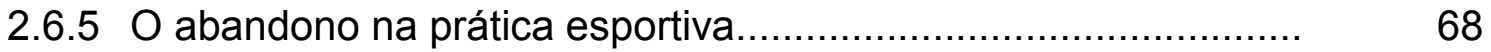

2.6.6 O papel do profissional na motivação dos praticantes................... 74

3 PROCEDIMENTOS METODOLÓGICOS .................................. 77

3.1 Natureza da pesquisa.............................................................

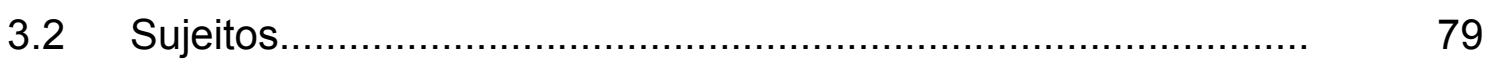

3.3 Técnica de coleta de dados.....................................................

3.4 Método de análise dos dados.................................................... 84

$4 \quad$ RESULTADOS E DISCUSSÃO...............................................

4.1 Características gerais dos sujeitos........................................... 87

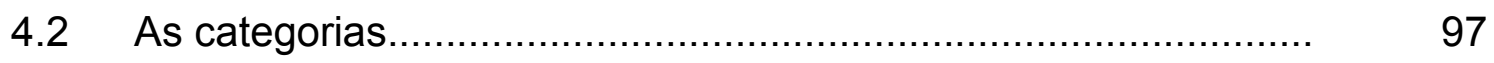

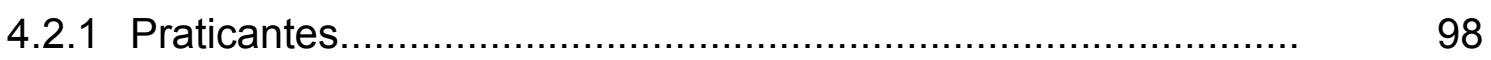

4.2.2 Professores................................................................... 145

4.2.3 Professores e Praticantes...................................................... 185

$5 \quad$ CONSIDERAÇÕES FINAIS.................................................... 189

6 REFERÊNCIAS BIBLIOGRÁFICAS......................................... 195

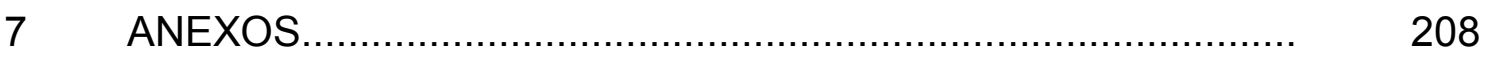




\section{LISTA DE QUADROS}

Página

QUADRO 1- Adaptado de Tubino (2002, p.66).

QUADRO 2 - As fases do desenvolvimento motor. Adaptado de Gallahue e Ozmun (2005, p.57).

QUADRO 3 - Adaptado de Nunomura (1998, p.66)...

QUADRO 4 - Diferenças entre Atividade GA e Esporte GA

QUADRO 5 - Faixas etárias em que são oferecidas GA como atividade esportiva extracurricular em escolas da Grande São Paulo...

QUADRO 6 - Faixa etária de praticantes de GA em colégios.

QUADRO 7 - Opinião dos professores sobre a faixa etária com maiores problemas de motivação para prática de GA

QUADRO 8 - Composição dos sujeitos.

QUADRO 9 - Perfil dos CGP.

QUADRO 10 - Perfil dos praticantes dos CGP.

QUADRO 11 - Resumo das características da infra-estrutura dos CGP.

QUADRO 12 - Perfil dos CPP

QUADRO 13 - Perfil dos praticantes dos CPP...

QUADRO 14 - Resumo das características da infra-estrutura dos CPP

QUADRO 15 - Perfil dos sujeitos "professores dos CGP (PGP).

QUADRO 16 - Resumo dos objetivos do programa de GA de acordo com as professoras dos CGP 
QUADRO 18 - Resumo dos objetivos do programa de GA de acordo com os professores dos CPP..................................................... 93

QUADRO 19 - Perfil dos sujeitos "praticantes" dos CGP (GP)............. 94

QUADRO 20 - Perfil dos sujeitos "praticantes" dos CPP (PP).............. 95

QUADRO 21 - Exemplo 1 de organização da apresentação dos dados.

QUADRO 22 - Exemplo 2 de organização da apresentação dos dados.

QUADRO 23 - Exemplo 3 de organização da apresentação dos dados.

QUADRO 24 - Exemplo 4 de organização da apresentação dos dados

QUADRO 25 - Resultados referentes aos motivos de escolha pela GA.

QUADRO 25a - Motivos intrínsecos de escolha pela GA - praticantes dos CGP ente 11 e 14 anos de idade

QUADRO 25b - Motivos intrínsecos de escolha pela GA - praticantes dos CPP entre 11 e 14 anos de idade.

QUADRO 25c - Motivos intrínsecos de escolha pela GA - praticantes dos CGP entre 15 e 17 anos de idade.

QUADRO 25d - Motivos extrínsecos de escolha pela GA - praticantes dos CGP entre 11 e 14 anos de idade.

QUADRO 25e - Motivos extrínsecos de escolha pela GA - praticantes dos CPP entre 11 e 14 anos de idade.

QUADRO 25f - Motivos extrínsecos de escolha pela GA - praticantes dos CGP entre 15 e 17 anos de idade.

QUADRO 25g - Motivos extrínsecos de escolha pela GA - praticantes dos CPP entre 15 e 17 anos de idade.

QUADRO 26 - Resultados referentes aos objetivos na GA.

QUADRO 26a - Objetivos diretamente relacionados à GA praticantes dos CGP entre 11 e 14 anos de idade. 
QUADRO 26b - Objetivos diretamente relacionados à GA praticantes dos CPP entre 11 e 14 anos de idade

QUADRO 26c - Objetivos diretamente relacionados à GA praticantes dos CGP entre 15 e 17 anos de idade.

QUADRO 26d - Objetivos diretamente relacionados à GA praticantes dos CPP entre 15 e 17 anos de idade.

QUADRO 26e - Objetivos indiretamente relacionados à GA praticantes dos CGP entre 11 e 14 anos de idade.

QUADRO 26f - Objetivos indiretamente relacionados à GA praticantes dos CPP entre 11 e 14 anos de idade.

QUADRO 26g - Objetivos indiretamente relacionados à GA praticantes dos CGP entre 15 e 17 anos de idade.

QUADRO 26h - Objetivos indiretamente relacionados à GA praticantes dos CPP entre 15 e 17 anos de idade.

QUADRO 27 - Resultados referentes aos motivos de permanência na GA.

QUADRO 27a - Motivos intrínsecos de permanência na GA praticantes dos CGP entre 11 e 14 anos de idade.

QUADRO 27b - Motivos intrínsecos de permanência na GA praticantes dos CPP entre 11 e 14 anos de idade.

QUADRO 27c - Motivos intrínsecos de permanência na GA praticantes dos CGP entre 15 e 17 anos de idade.

QUADRO 27d - Motivos intrínsecos de permanência na GA praticantes dos CPP entre 15 e 17 anos de idade

QUADRO 27e - Motivos extrínsecos de permanência na GA praticantes dos CGP entre 11 e 14 anos de idade.

QUADRO $27 f$ - Motivos extrínsecos de permanência na GA praticantes dos CPP entre 11 e 14 anos de idade.

QUADRO 27g - Motivos extrínsecos de permanência na GA praticantes dos CGP entre 15 e 17 anos de idade.

QUADRO 27h - Motivos extrínsecos de permanência na GA praticantes dos CPP entre 15 e 17 anos de idade. 
QUADRO 28 - Resultados sobre o tempo que a praticante pretende se dedicar à GA......

QUADRO 28a - Tempo de dedicação à GA pelas praticantes dos CGP entre 11 e 14 anos de idade.

QUADRO 28b - Tempo de dedicação à GA pelas praticantes dos CPP entre 11 e 14 anos de idade.

QUADRO 28c - Tempo de dedicação à GA pelas praticantes dos CGP entre 15 e 17 anos de idade.

QUADRO 28d - Tempo de dedicação à GA pelas praticantes dos CPP entre 15 e 17 anos de idade.

QUADRO 29 - Resultados sobre os motivos de afastamento temporário da GA.

QUADRO 29a - Motivos intrínsecos de afastamento temporário da GA - praticantes dos CGP entre 15 e 17 anos de idade.

QUADRO 29b - Motivos extrínsecos de afastamento temporário da GA - praticantes dos CGP entre 11 e 14 anos de idade.

QUADRO 29c - Motivos extrínsecos de afastamento temporário da GA - praticantes dos CPP entre 11 e 14 anos de idade.

QUADRO 29d - Motivos extrínsecos de afastamento temporário da GA - praticantes dos CGP entre 15 e 17 anos de idade.

QUADRO $29 f$ - Motivos extrínsecos de afastamento temporário da GA - praticantes dos CPP entre 15 e 17 anos de idade.

QUADRO 30 - Resultados sobre os motivos de retorno após afastamento temporário.

QUADRO 30a - Motivos intrínsecos de retorno após afastamento temporário - praticantes dos CPP entre 11 e 14 anos de idade.

QUADRO 30b - Motivos intrínsecos de retorno após afastamento temporário - praticantes dos CGP de 15 à 17 anos de idade.

QUADRO 30c - Motivos extrínsecos de retorno após afastamento temporário - praticantes dos CGP entre 11 e 14 anos de idade.

QUADRO 30d - Motivos extrínsecos de retorno após afastamento temporário - praticantes dos CPP entre 11 e 14 anos de idade. 
QUADRO 30e - Motivos extrínsecos de retorno após afastamento temporário - praticantes dos CGP entre 15 e 17 anos de idade.

QUADRO 30f - Motivos extrínsecos de retorno após afastamento temporário - praticantes dos CPP entre 15 e 17 anos de idade.

QUADRO 31 - Resultados referentes a relação entre nível de habilidade e motivação.

QUADRO 31a - Relação entre nível de habilidade e motivação praticantes dos CGP entre 11 e 14 anos de idade.

QUADRO 31b - Relação entre nível de habilidade e motivação praticantes dos CPP entre 11 e 14 anos de idade.

QUADRO 31c - Relação entre nível de habilidade e motivação praticantes dos CGP entre 15 e 17 anos de idade.

QUADRO 31d - Relação entre nível de habilidade e motivação praticantes dos CPP entre 15 e 17 anos de idade.

QUADRO 32 - Resultados sobre as preferências de atividades na GA.

QUADRO 32a - Preferências por aparelhos em geral pelas praticantes dos CGP entre 11 e 14 anos de idade.

QUADRO 32b - Preferências por aparelhos em geral pelas praticantes dos CPP entre 11 e 14 anos de idade.

QUADRO 32c - Preferências por aparelhos em geral pelas praticantes dos CGP entre 15 e 17 anos de idade.

QUADRO 32d - Preferências pelas características dos aparelhos/movimentos pelas praticantes dos CGP entre 11 e 14 anos de idade.

QUADRO 32e - Preferências pelas características dos aparelhos/movimentos pelas praticantes dos CPP entre 11 e 14 anos de idade

QUADRO $32 f$ - Preferências pelas características dos aparelhos/movimentos pelas praticantes dos CGP entre 15 e 17 anos de idade. 
QUADRO $32 \mathrm{~g}$ - Preferências pelas características dos aparelhos/movimentos pelas praticantes dos CPP entre 15 e 17 anos de idade.

QUADRO 32h - Preferência por treinamento para participação em eventos pelas praticantes dos CGP entre 11 e 14 anos de idade.

QUADRO 32i - Preferência por treinamento para participação em eventos pelas praticantes dos CGP entre 15 e 17 anos de idade.

QUADRO 32j - Preferência por treinamento para participação em eventos pelas praticantes dos CPP entre 15 e 17 anos de idade.

QUADRO 32I - Preferência por outras atividades pelas praticantes dos CGP entre 11 e 14 anos de idade.

QUADRO $32 \mathrm{~m}$ - Preferência por outras atividades pelas praticantes dos CGP entre 15 e 17 anos de idade.

QUADRO 33 - Resultados sobre as atividades menos apreciadas nas aulas de GA.

QUADRO 33a - Atividades menos apreciadas pelas praticantes dos CGP entre 11 e 14 anos de idade.

QUADRO 33b - Atividades menos apreciadas pelas praticantes dos CPP entre 11 e 14 anos de idade.

QUADRO 33c - Atividades menos apreciadas pelas praticantes dos CGP entre 15 e 17 anos de idade.

QUADRO 33d - Atividades menos apreciadas pelas praticantes dos CPP entre 15 e 17 anos de idade.

QUADRO 34 - Resultados sobre as estratégias para lidar com as atividades menos apreciadas.

QUADRO 34a - Estratégias para lidar com as atividades menos apreciadas - praticantes dos CGP entre 11 e 14 anos de idade.

QUADRO 34b - Estratégias para lidar com as atividades menos apreciadas - praticantes dos CPP entre 11 e 14 anos de idade.

QUADRO 34c - Estratégias para lidar com as atividades menos apreciadas - praticantes dos CGP entre 15 e 17 anos de idade. 
QUADRO 34d - Estratégias para lidar com as atividades menos apreciadas - praticantes dos CPP entre 15 e 17 anos de idade.

QUADRO 35 - Resultados sobre a motivação para participar em eventos.

QUADRO 35a - Motivação para competir - praticantes dos CGP entre 11 e 14 anos de idade.

QUADRO 35b - Motivação para competir - praticantes dos CPP entre 11 e 14 anos de idade.

QUADRO 35c - Motivação para competir - praticantes dos CGP entre 15 e 17 anos de idade.

QUADRO 35d - Motivação para participar de festivais - praticantes dos CGP entre 11 e 14 anos de idade.

QUADRO 35e - Motivação para participar de festivais - praticantes dos CPP entre 11 e 14 anos de idade.

QUADRO 35f - Motivação para participar de apresentações em grupo - praticantes dos CGP entre 11 e 14 anos de idade.

QUADRO 35g - Motivação para participar de apresentações em grupo - praticantes dos CGP entre 15 e 17 anos de idade.

QUADRO 35h - Motivação para participar de apresentações em grupo - praticantes dos CPP entre 15 e 17 anos de idade.

QUADRO 36 - Resultados sobre a opinião das praticantes em relação aos professores.

QUADRO 36a - Opinião das praticantes dos CGP entre 11 e 14 anos de idade em relação às características pessoais positivas de seus professores.

QUADRO 36b - Opinião das praticantes dos CPP entre 11 e 14 anos de idade em relação às características pessoais positivas de seus professores.

QUADRO 36c - Opinião das praticantes dos CGP entre 15 e 17 anos de idade em relação às características pessoais positivas de seus professores. 
QUADRO 36d - Opinião das praticantes dos CPP entre 15 e 17 anos de idade em relação às características pessoais positivas de seus professores.

QUADRO 36e - Opinião das praticantes dos CGP entre 11 e 14 anos de idade em relação às características pessoais negativas de seus professores.

QUADRO 36f - Opinião das praticantes dos CGP entre 15 e 17 anos de idade em relação às características pessoais negativas de seus professores.

QUADRO 36g - Opinião das praticantes dos CGP entre 11 e 14 anos de idade em relação às características pedagógicas positivas de seus professores.

QUADRO 36h - Opinião das alunas dos CPP entre 11 e 14 anos de idade em relação às características pedagógicas positivas de seus professores.

QUADRO 36i - Opinião das praticantes dos CGP entre 15 e 17 anos de idade em relação às características pedagógicas positivas de seus professores.

QUADRO 36j - Opinião das praticantes dos CGP entre 11 e 14 anos de idade em relação às características pedagógicas negativas de seus professores.

QUADRO 36I - Opinião das praticantes dos CPP entre 11 e 14 anos de idade em relação às características pedagógicas negativas de seus professores

QUADRO 36m - Opinião das praticantes dos CGP entre 15 e 17 anos de idade em relação às características pedagógicas negativas de seus professores.

QUADRO 37 - Resultados referentes a opinião do professor sobre a idade ideal para iniciar a prática de GA na escola.

QUADRO 37a - Opinião dos PGP sobre a idade ideal para iniciar a prática de GA na escola..... 
QUADRO 37b - Opinião dos PPP sobre a idade ideal para iniciar a prática de GA na escola.

QUADRO 38 - Resultados referentes a opinião do professor sobre a idade limite para iniciar a prática de GA na escola.

QUADRO 38a - Opinião dos PGP sobre a idade limite para iniciar a prática de GA na escola.

QUADRO 38b - Opinião dos PPP sobre a idade limite para iniciar a prática de GA na escola.

QUADRO 39 - Resultados referentes aos problemas associados à motivação para prática de GA na escola.

QUADRO 39a - Fatores intrínsecos relatados pelos PGP associados à motivação para prática de GA na escola..

QUADRO 39b - Fatores intrínsecos relatados pelos PPP associados à motivação para prática de GA na escola.

QUADRO 39c - Fatores extrínsecos relatados pelos PGP associados à motivação para prática de GA na escola.

QUADRO 39d - Fatores extrínsecos relatados pelos PPP associados à motivação para prática de GA na escola.

QUADRO 40 - Resultados referentes aos fatores que favorecem a motivação.

QUADRO 40a - Fatores que favorecem a motivação relatados por PGP.

QUADRO 41 - Resultados referentes ao interesse dos professores pelos motivos de desistência dos praticantes.

QUADRO 41a - Interesse dos PGP pelos motivos de desistência dos praticantes.

QUADRO 41b - Interesse dos PPP pelos motivos de desistência dos praticantes.

QUADRO 42 - Resultados referentes aos motivos de desistência.

QUADRO 42a - Fatores intrínsecos citados por PGP relacionados aos motivos de desistência. 
QUADRO 42b - Fatores extrínsecos citados por PGP relacionados aos motivos de desistência.

QUADRO 42c - Fatores extrínsecos citados por PPP relacionados aos motivos de desistência.

QUADRO 43 - Resultados sobre atitudes e estratégias em relação aos motivos de desistência.

QUADRO 43a - Atitudes e estratégias em relação aos motivos de desistência relatados por PGP.

QUADRO 43b - Atitudes e estratégias em relação aos motivos de desistência relatados por PPP.

QUADRO 44 - Resultados referentes as estratégias para atrair praticantes.

QUADRO 44a - Divulgação da aula para atrair praticantes relatados por PGP.

QUADRO 44b - Divulgação da aula para atrair praticantes relatados por PPP.

QUADRO 44C - Divulgação da modalidade para atrair praticantes relatados por $\mathrm{PGP}$.

QUADRO 44d - Divulgação da modalidade para atrair praticantes relatados por PPP.

QUADRO 45 - Resultados referentes as estratégias para manter a motivação para prática de GA

QUADRO 45a - Estratégias extra aula para manter a motivação para prática de GA relatada por PGP.

QUADRO 45b - Estratégias extra aula para manter a motivação para prática de GA relatada por PPP.

QUADRO 45c - Estratégias durante a aula para manter a motivação para prática de GA relatada por PGP.

QUADRO 45d - Estratégias durante a aula para manter a motivação para prática de GA relatada por PPP.

QUADRO 46 - Resultados sobre a relação entre nível de habilidade e motivação. 
QUADRO 46a - Resultados sobre nível de habilidade e motivação relatado por $\mathrm{PGP}$

QUADRO 46b - Resultados sobre nível de habilidade e motivação relatado por PPP.

QUADRO 47 - Resultados referentes a opinião dos professores sobre o motivo que levaram as praticantes para prática de GA

QUADRO 47a - Opinião dos PGP sobre os motivos intrínsecos que trazem as praticantes para prática de GA.....

QUADRO 47b - Opinião dos PPP sobre os motivos intrínsecos que trazem as praticantes para prática de GA

QUADRO 47c - Opinião dos PGP sobre os motivos extrínsecos que trazem as praticantes para prática de $\mathrm{GA}$

QUADRO 47d - Opinião dos PPP sobre os motivos extrínsecos que trazem as praticantes para prática de GA

QUADRO 48 - Resultados referentes a opinião dos professores sobre o objetivo das praticantes com a prática de GA.

QUADRO 48a - Opinião dos PGP sobre o objetivo das praticantes com a prática de $\mathrm{GA}$.

QUADRO 48b - Opinião dos PPP sobre o objetivo das praticantes com a prática de GA.

QUADRO 49 - Resultados referentes a opinião dos professores sobre os motivos de permanência na prática de GA.

QUADRO 49a - Opinião dos PGP sobre os motivos intrínsecos de permanência na prática de GA

QUADRO 49b - Opinião dos PPP sobre os motivos intrínsecos de permanência na prática de GA.

QUADRO 49c - Opinião dos PGP sobre os motivos extrínsecos de permanência na prática de GA.

QUADRO 49d - Opinião dos PPP sobre os motivos extrínsecos de permanência na prática de GA

QUADRO 50 - Resultados referentes a opinião dos professores sobre as atividades preferidas nas aulas pelas praticantes. 
QUADRO 50a - Opinião dos PGP sobre as atividades preferidas nas aulas pelas praticantes.

QUADRO 50b - Opinião dos PPP sobre as atividades preferidas nas aulas pelas praticantes.

QUADRO 51 - Resultados referentes a opinião dos professores sobre as atividades menos apreciadas pelas praticantes

QUADRO 51a - Opinião dos PGP sobre as atividades menos apreciadas pelas praticantes

QUADRO 51b - Opinião dos PPP sobre as atividades menos apreciadas pelas praticantes.

QUADRO 52 - Resultados referentes as estratégias para lidar com as atividades menos apreciadas pelas praticantes.

QUADRO 52a - Estratégias dos PGP para lidar com as atividades menos apreciadas pelas praticantes

QUADRO 52b - Estratégias dos PPP para lidar com as atividades menos apreciadas pelas praticantes

QUADRO 53 - Resultados referentes à opinião dos professores sobre a motivação para participação em eventos

QUADRO 53a - Opinião dos PGP sobre a motivação para competir.....

QUADRO 53b - Opinião dos PPP sobre a motivação para competir.....

QUADRO 53c - Opinião dos PGP sobre a motivação para participação em festivais

QUADRO 53d - Opinião dos PPP sobre a motivação para participação em festivais.

QUADRO 53e - Opinião dos PGP sobre a motivação para participação em apresentações em grupo.

QUADRO 53f - Opinião dos PPP sobre a motivação para participação em apresentações em grupo

QUADRO 53g - Opinião dos PPP sobre a motivação para participação em eventos em geral. 


\section{LISTA DE FIGURAS}

Página

FIGURA 1 - Adaptado de Sawasato e Castro (2006, p.111)...................

FIGURA 2 - Modelo de motivação interacional de indivíduo-situação adaptado de Weinberg e Gould (2001, p.75)...... 


\section{LISTA DE SIGLAS, ABREVIAÇÕES E SÍMBOLOS}

$\begin{array}{lll}\text { AC } & - & \text { Análise de Conteúdo } \\ \text { CBG } & - & \text { Confederação Brasileira de Ginástica } \\ \text { CGP } & - & \text { Colégios de grande porte } \\ \text { CPP } & - & \text { Colégios de pequeno porte } \\ \text { GA } & - & \text { Ginástica Artística } \\ \text { GD } & - & \text { Ginástica Desenvolvimentista } \\ \text { GE } & - & \text { Ginástica Educacional } \\ \text { GP } & - & \text { Praticantes dos colégios de grande porte } \\ \text { PP } & - & \text { Praticantes dos colégios de pequeno porte } \\ \text { PGP } & - & \text { Professores dos colégios de grande porte } \\ \text { PPP } & - & \text { Professores dos colégios de pequeno porte } \\ \text { RRAMM } & - & \text { Redução dos Riscos de Adoecer e Morrer na Maturidade } \\ \text { UNESCO } & - & \text { Organização das Nações Unidas }\end{array}$




\section{LISTA DE ANEXOS}

Página

ANEXO I - Formulário entregue para professores de escolas que possuem GA em seu quadro de atividades extracurriculares

ANEXO II - Carta de esclarecimento apresentada aos coordenadores dos colégios participantes..

ANEXO III - Roteiro de entrevista.

ANEXO IV - Aprovação pelo Comitê de Ética da EEFEUSP 
RESUMO

\title{
MOTIVAÇÃO E GINÁSTICA ARTÍSTICA FORMATIVA NO CONTEXTO EXTRACURRICULAR
}

\author{
Autora: Priscila Lopes \\ Orientadora: Prof ${ }^{\mathrm{a}}$. Dr ${ }^{\mathrm{a}}$. Myrian Nunomura
}

Até há pouco tempo, a Ginástica Artística (GA) era uma modalidade esportiva conhecida e praticada por uma minoria no Brasil, situação que vem sendo ligeiramente alterada devido a popularidade das conquistas internacionais de nossos ginastas. Este fato parece estar aumentando o interesse das crianças pela GA gradativamente. A motivação é tema de destaque nos estudos da área do Esporte. Assim, o conhecimento e a compreensão de aspectos que motivam a prática de GA formativa podem auxiliar os profissionais a desenvolver um trabalho cada vez mais atraente e estimulador, bem como tornar a experiência mais significativa e prazerosa aos praticantes, minimizar problemas de desistências e incentivar a massificação da modalidade. A partir de entrevistas com meninas praticantes entre 11 e 17 anos de idade e seus respectivos professores, investigamos a motivação dessas jovens para a prática da GA nas escolas e as estratégias dos professores para estimular e manter esse interesse. Verificamos que há um conjunto de motivos que incentivam o ingresso e a permanência na modalidade, o qual tem origem intrínseca ou extrínseca. As estratégias motivacionais dos professores se mostraram relativamente eficientes. Entretanto, sugerimos que fatores motivacionais 
intrínsecos e extrínsecos sejam considerados e que a comunicação entre alunas e professores seja intensificada a fim de atender às necessidades e expectativas das praticantes.

Palavras chave: Ginástica Artística, Motivação, Esporte na escola 


\title{
ABSTRACT \\ MOTIVATION AND FORMATIVE ARTISTIC GYMNASTICS IN THE EXTRACURRICULAR CONTEXT
}

\author{
Author: Priscila Lopes \\ Adviser: Prof ${ }^{\mathrm{a}}$. Dr ${ }^{\mathrm{a}}$. Myrian Nunomura
}

Up to now, Artistic Gymnastics (AG) was a sport only known and practiced by only a small minority of Brazilians, this situation has been changing by the popularity of our athletes in international Olympics. In fact, the interests of many children in AG have been increasing steadily. Motivation is a highlighted issue within Sports related studies. Knowledge and understanding of motivation in AG may help professionals to develop a better and more stimulating program and make this experience even more meaningful and joyful to the learners, minimize dropouts and increase the popularity of this activity. We interviewed coaches and their female students ageing from 11 to 17 years and we analyzed the girls motivation to practice AG in the schools and the strategy used by coaches to stimulate and to maintain their interest. We verified that there are many reasons that work for the starting and remaining in this particular sport, which may have an intrinsic or extrinsic origins. Motivational strategies used by coaches were relatively effective. However, we suggest that the intrinsic and extrinsic motivational factors would be considered and the coach-practitioner communication be intensified in order to meet the needs and expectations of the learners.

Key words: Artistic Gymnastic, Motivation, Sports in school. 